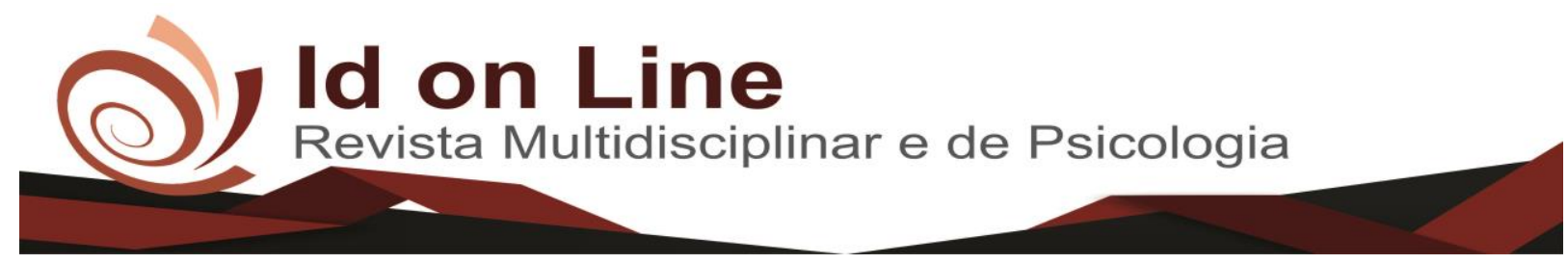

Artigo

\title{
De "Peste Gay" à Supremacia da AIDS entre Heterossexuais no Brasil
}

\author{
Pedro Walisson Gomes Feitosa ${ }^{1}$, Ítalo Gonçalves Pita de Oliveira ${ }^{2}$, Maria Andrezza Gomes Maia ${ }^{3}$, \\ Maria Vitória Filgueira Martins ${ }^{4}$, Elisa Hellen Cruz Rodrigues ${ }^{5}$, Esther Barbosa Gonçalves Felix ${ }^{6,}$ \\ Estelita Lima Cândido ${ }^{7}$, Paulo Renato Alves Firmino ${ }^{8}$
}

\begin{abstract}
Resumo: O objetivo do estudo foi descrever o cenário epidemiológico e a evolução das ocorrências da Síndrome da Imunodeficiência Adquirida no Brasil entre os anos de 2010 e 2016, consoante as variáveis sexo, região de incidência e categoria de exposição, com base nos boletins emitidos pelo Ministério da Saúde do Brasil. Os dados foram analisados através do programa Bioestat 5.0. Durante estes anos, foram notificados $125.806 \mathrm{casos}$ em homens e 62.920 casos em mulheres. Entre os homens verifica-se que mais de $75 \%$ dos casos foram adquiridos por transmissão sexual (predominando a exposição heterossexual) e, entre as mulheres, mais de $85 \%$. O artigo constata a representação epidemiológica do estereótipo da síndrome hodiernamente: homem, heterossexual, habitante dos grandes centros urbanos. Logo, as altas taxas anuais de infecção sugerem que o modo de abordar a doença precisa ser ampliado.
\end{abstract}

Palavras-chave: HIV; AIDS; Epidemiologia;

\section{From "gay plague" to AIDS supremacy among heterosexuals in Brazil}

\begin{abstract}
The objective of the study was to describe the epidemiological scenario and the evolution of the occurrences of Acquired Immunodeficiency Syndrome in Brazil between the years 2010 and 2016, according to the variables gender, incidence region and exposure category, based on the bulletins issued by the Ministry of Health of Brazil. The data were analyzed through the program Bioestat 5.0. During these years, 125,806 cases were reported in men and 62,920 cases in women. Among men it is verified that more than $75 \%$ of the cases had sexual transmission (predominating heterosexual exposure) and, among the women, more than $85 \%$. The article verifies the epidemiological representation of the stereotype of the syndrome nowadays: man, heterosexual, inhabitant of large urban centers. Therefore, the high annual rates of infection suggest that the approach to the disease needs to be expanded.
\end{abstract}

Keywords: HIV; AIDS; Epidemiology;

\footnotetext{
${ }^{1}$ Acadêmico de Medicina da Universidade Federal do Cariri, UFCA, Brasil. gomesfeitosa.walisson@ outlook.com

${ }^{2}$ Acadêmico de Medicina da Universidade Federal do Cariri, UFCA, Brasil. italopita@ hotmail.com

${ }^{3}$ Acadêmica de Medicina da Universidade Federal do Cariri, UFCA, Brasil. andrezzamaia12@gmail.com

${ }^{4}$ Acadêmica de Medicina da Universidade Federal do Cariri, UFCA, Brasil. vitoriafilgueira22@ gmail.com

${ }^{5}$ Acadêmica de Medicina da Universidade Federal do Cariri, UFCA, Brasil. elisa.cruz24@ gmail.com

${ }^{6}$ Acadêmica de Medicina da Universidade Federal do Cariri, UFCA, Brasil. esther.barbosa.g@gmail.com

${ }^{7}$ Professora adjunta da Universidade Federal do Cariri, UFCA. Pós-Doutorado em Ciências da Saúde. Docente permanente do Programa de Pós-Graduação em Desenvolvimento Regional Sustentável da UFCA e do Mestrado profissional em Formação em Saúde da Família da RENASF/URCA, Brasil. estelita.lima@ufca.edu.br

${ }^{8}$ Professor de Estatística da Universidade Federal do Cariri, UFCA. Docente permanente do Programa de Pós-Graduação em Desenvolvimento Regional Sustentável da UFCA e colaborador do Programa de Pós-Graduação em Biometria e Estatística Aplicada da UFRPE, Brasil. paulo.firmino@ufca.edu.br.
} 


\section{Introdução}

Segundo o Programa Nacional de Aids, a Síndrome da Imunodeficiência Adquirida começou a ser observada na metade do século XX. Os relatos contam que a doença surgiu na África Central e, provavelmente, pela mutação do vírus do macaco. Algumas experiências comprovam que o elo perdido na passagem dos primatas para o homem parece estar relacionado com a questão da manipulação de carnes de chimpanzés infectados na África. A doença, então levada para pequenas comunidades da região central, disseminou-se pelo mundo todo com a globalização (Brasil, 2006).

A Aids é causada pela infecção pelo Vírus da Imunodeficiência Humana (HIV), caracterizada por períodos de latência prolongados, destruindo células do sistema imune. O início da infecção pode ser confundido com um processo virótico de vias aéreas, da qual seguese um longo período de latência, em que o paciente se apresenta assintomático (Greco et al. 2016). A síndrome é definida pelo aparecimento de infecções oportunistas. O vírus é transmitido através do ato sexual, por via sanguínea e por via vertical. Não é comprovada a transmissão por meio de suor, urina, vômitos ou por saliva. Há relatos de transmissão por meio de inseminação artificial, transplante de órgãos e tratamento dentário com profissional infectado (BRASIL,_2013).

Após mais de 30 anos de sua identificação, a Aids é considerada a maior pandemia do século XX. A maioria das pessoas infectadas vivem em países subdesenvolvidos, principalmente na África Subsaariana. A doença foi investigada e descrita pela primeira vez em 1981. Inicialmente foi diagnosticada em indivíduos masculinos de orientação homo ou bissexual; no entanto, à posteriori, foi identificada em heterossexuais, mulheres, usuários de drogas injetáveis (UDI) e hemofílicos (GRECO et al. 2016).

Além do impacto das ocorrências da síndrome na saúde, o HIV funciona como uma lente que amplia os males da sociedade e as deficiências dos sistemas sociais. Nesse viés, as repostas às epidemias promovidas pelos governos dos países têm proporcionado uma oportunidade para fortalecer o tecido social, melhorar a justiça social e reforçar os sistemas que prestam serviços essenciais aos segmentos mais vulneráveis das comunidades. É necessário se obter o equilíbrio entre a intensificação do trabalho nos países mais afetados e a identificação de outros contextos, como os grandes centros urbanos, onde o impacto do HIV está afetando comunidades específicas (BRASIL, 2010). 
Inicialmente, no Brasil, a epidemia era marcadamente caracterizada pela transmissão sexual homo ou bissexual. Em seguida, deu-se notória importância à transmissão sanguínea, principalmente em UDIs. Posteriormente, houve um aumento da transmissão sexual da doença por indivíduos heterossexuais, bem como do número de mulheres infectadas (ANJOS et al 2007). As estimativas são de que aproximadamente 718 mil indivíduos vivam com o HIV/AIDS no Brasil, porém apenas $80 \%$ conhecem seu diagnóstico (BRASIL, 2013).

Outrossim, as estatísticas têm comprovado que a doença se intensificou, em especial no que se refere à feminilização. Isso se deve, particularmente, à maior exposição da mulher frente ao baixo poder de decisão destas em questões que envolvam sua vida sexual e reprodutiva (NASCIMENTO et al 2005).

O HIV e a Aids fazem parte da Lista Nacional de Notificação Compulsória de doenças (Portaria $\mathrm{n}^{\circ}$ 204, de 17 de fevereiro de 2016). Todavia, a subnotificação de casos no Sistema de Informação de Agravos de Notificação (SINAN) traz importantes implicações para a resposta ao HIV/Aids, posto que permanecem desconhecidas informações importantes no âmbito da epidemiologia, tais como número total de casos, comportamentos e vulnerabilidades. Além disso, a ausência de registro pode ter reflexo na programação orçamentária do Poder Público, comprometendo a racionalização dos recursos para o fornecimento contínuo de medicamentos (BRASIL, 2016).

Assim sendo, o presente artigo tem por objetivo descrever o cenário epidemiológico e a evolução das ocorrências da Síndrome da Imunodeficiência Adquirida entre os anos de 2000 a 2016 no Brasil.

\section{Metodologia}

Trata-se de um estudo documental, com base na consulta aos boletins epidemiológicos divulgados pelo Ministério da Saúde referentes ao período de 2010-2016. Esses documentos são elaborados com dados provenientes do Sistema de Informação de Agravos de Notificação (SINAN).

A incidência de Aids foi analisada no referido período segundo o gênero, espaço e categoria de exposição. Os dados foram submetidos ao tratamento estatístico, através do programa Bioestat 5.0, que permitiu realizar comparação de médias do número de casos por categoria de exposição ao nível de significância de 0,05 . 


\section{Resultados}

Na Tabela 1 são apresentados casos de Aids notificados no SINAN entre 2010 e 2016, segundo sexo e categoria de exposição. Durante estes anos, foram notificados 125.806 casos em homens e 62.920 casos em mulheres, sendo, portanto, a razão entre a quantidade de casos nos sexos masculino e feminino equivalente a aproximadamente 2. Entre os homens, verificase que mais de $75 \%$ dos casos tiveram transmissão sexual (predominando a exposição heterossexual) (Tabela 1). Entre as mulheres, observa-se que mais de $85 \%$ dos casos tiveram transmissão sexual (predominando a exposição heterossexual). Quanto à transmissão vertical, foram notificados $1.618(1,3 \%)$ de casos do sexo masculino e $1.639(2,6 \%)$ do sexo feminino. Em relação à acidentes no trabalho, 5 casos foram notificados em cada sexo no período analisado. Por transfusão sanguínea, 20 e 19 casos entre homens e mulheres, respectivamente. 
Tabela 1: Notificações, em números absolutos (N) e relativos (\%), de Aids no Sinan em indivíduos, de acordo com o sexo, o ano de diagnóstico e a categoria de exposição. Brasil, 2010-2016.

\begin{tabular}{|c|c|c|c|c|c|c|c|c|c|c|c|c|c|c|c|c|c|}
\hline \multirow[t]{3}{*}{ Sexo } & \multirow{3}{*}{$\begin{array}{l}\text { Categoria de } \\
\text { exposição }\end{array}$} & \multicolumn{14}{|c|}{ Ano } & \multirow{2}{*}{\multicolumn{2}{|c|}{$\begin{array}{l}\text { Total do } \\
\text { período }\end{array}$}} \\
\hline & & \multicolumn{2}{|c|}{2010} & \multicolumn{2}{|c|}{2011} & \multicolumn{2}{|c|}{2012} & \multicolumn{2}{|c|}{2013} & \multicolumn{2}{|c|}{2014} & \multicolumn{2}{|c|}{2015} & \multicolumn{2}{|c|}{2016} & & \\
\hline & & $\mathbf{N}$ & $\%$ & $\mathbf{N}$ & $\%$ & $\mathbf{N}$ & $\%$ & $\mathbf{N}$ & $\%$ & $\mathbf{N}$ & $\%$ & $\mathbf{N}$ & $\%$ & $\mathbf{N}$ & $\%$ & $\mathbf{N}$ & $\%$ \\
\hline \multirow{11}{*}{ 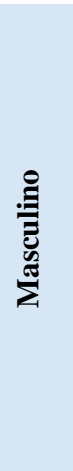 } & Homossexual & 2780 & 27,6 & 4097 & 29,4 & 4914 & 32,5 & 5557 & 33,7 & 5744 & 35,8 & 5452 & 36,4 & 5861 & 38,8 & 34405 & 27,3 \\
\hline & Bissexual & 997 & 9,9 & 1369 & 9,8 & 1431 & 9,5 & 1541 & 9,3 & 1436 & 8,9 & 1329 & 8,9 & 1314 & 8,7 & 9417 & 7,5 \\
\hline & Heterossexual & 5448 & 54,0 & 7405 & 53,1 & 7827 & 51,6 & 8398 & 50,9 & 7976 & 49,7 & 7394 & 49,3 & 7190 & 47,6 & 51638 & 41 \\
\hline & UDI & 691 & 6,8 & 826 & 5,9 & 708 & 4,8 & 745 & 4,5 & 642 & 4,0 & 566 & 3,8 & 487 & 3,2 & 4665 & 3,7 \\
\hline & Hemofílico & 5 & 0,0 & 6 & 0,0 & 6 & 0,0 & 6 & 0,0 & 6 & 0,0 & 9 & 0,1 & 4 & 0,0 & 42 & $\mathbf{0 , 0}$ \\
\hline & Transfusão & 4 & 0,0 & 2 & 0,0 & 5 & 0,0 & 1 & 0,0 & 2 & 0,0 & 4 & 0,0 & 2 & 0,0 & 20 & $\mathbf{0 , 0}$ \\
\hline & $\begin{array}{l}\text { Acidente } \\
\text { trabalho }\end{array}$ & 0 & 0,0 & 1 & 0,0 & 1 & 0,0 & 3 & 0,0 & 0 & 0,0 & 0 & 0,0 & 0 & 0,0 & 5 & $\mathbf{0 , 0}$ \\
\hline & $\begin{array}{l}\text { Transmissão } \\
\text { Vertical }\end{array}$ & 159 & 1,5 & 233 & 1,7 & 248 & 1,6 & 247 & 1,5 & 248 & 1,5 & 232 & 1,5 & 251 & 1,4 & 1618 & 1,3 \\
\hline & Subtotal & 10084 & 80,2 & 13939 & 80,4 & 15140 & 81,6 & 16498 & 81,5 & 16054 & 80,9 & 14986 & 80,3 & 15109 & 81,6 & 101810 & 80,9 \\
\hline & Ignorado & 2481 & 19,8 & 3399 & 19,6 & 3509 & 18,4 & 3750 & 18,5 & 3787 & 19,1 & 3674 & 19,7 & 3396 & 18,4 & 23996 & 19,1 \\
\hline & Total & 12.565 & 100,0 & 17.338 & 100,0 & 18.649 & 100,0 & 20.248 & 100,0 & 19.841 & 100,0 & 18.660 & 100,0 & 18.505 & 100,0 & 125.806 & 100,0 \\
\hline \multirow{11}{*}{ 思 } & Homossexual & 93 & 1,5 & 121 & 1,4 & 146 & 1,6 & 141 & 1,5 & 105 & 1,2 & 126 & 1,6 & 130 & 1,8 & 862 & 1,4 \\
\hline & Bissexual & 42 & 0,7 & 54 & 0,6 & 37 & 0,4 & 60 & 0,6 & 50 & 0,6 & 55 & 0,7 & 62 & 0,9 & 360 & 0,6 \\
\hline & Heterossexual & 5770 & 92,2 & 7864 & 92,6 & 8362 & 92,9 & 8692 & 93,0 & 8208 & 93,4 & 7136 & 92,8 & 6633 & 92,7 & 52665 & 83,7 \\
\hline & UDI & 155 & 2,5 & 210 & 2,5 & 198 & 2,2 & 183 & 2,0 & 163 & 1,9 & 150 & 1,9 & 121 & 1,7 & 1180 & 1,9 \\
\hline & Transfusão & 2 & 0,0 & 5 & 0,1 & 4 & 0,0 & 3 & 0,0 & 2 & 0,0 & 1 & 0,0 & 2 & 0,0 & 19 & $\mathbf{0 , 0}$ \\
\hline & $\begin{array}{l}\text { Acidente } \\
\text { trabalho }\end{array}$ & 0 & 0,0 & 3 & 0,0 & 1 & 0,0 & 1 & 0,0 & 0 & 0,0 & 0 & 0,0 & 0 & 0,0 & 5 & $\mathbf{0 , 0}$ \\
\hline & $\begin{array}{l}\text { Transmissão } \\
\text { Vertical }\end{array}$ & 198 & 3,2 & 237 & 2,8 & 257 & 2,9 & 264 & 2,8 & 253 & 2,9 & 221 & 2,9 & 209 & 2,9 & 1639 & 2,6 \\
\hline & Subtotal & 6260 & 90,4 & 8494 & 91,5 & 9005 & 91,0 & 9344 & 90,1 & 8783 & 89,9 & 7689 & 88,7 & 7157 & 88,7 & 56732 & 90,2 \\
\hline & Ignorado & 666 & 9,6 & 787 & 9,5 & 894 & 9,0 & 1023 & 9,9 & 986 & 10,1 & 978 & 11,3 & 856 & 11,3 & 6190 & 9,8 \\
\hline & Total & 6.926 & 100,0 & 9.281 & 100,0 & 9.899 & 100,0 & 10.367 & 100,0 & 9.769 & 100,0 & 8.667 & 100,0 & 8.013 & 100,0 & 62.926 & 100,0 \\
\hline & tal geral do ano & 19.491 & - & 26.691 & - & 28.548 & - & 30.615 & - & 29.610 & - & 27.327 & - & 26.518 & - & 188.732 & - \\
\hline
\end{tabular}

FONTE: Boletins epidemiológicos,2017. 
Tabela 2: Comparação do número médio de casos por categoria de exposição nos sexos masculino e feminino. Brasil, 2010-2016.

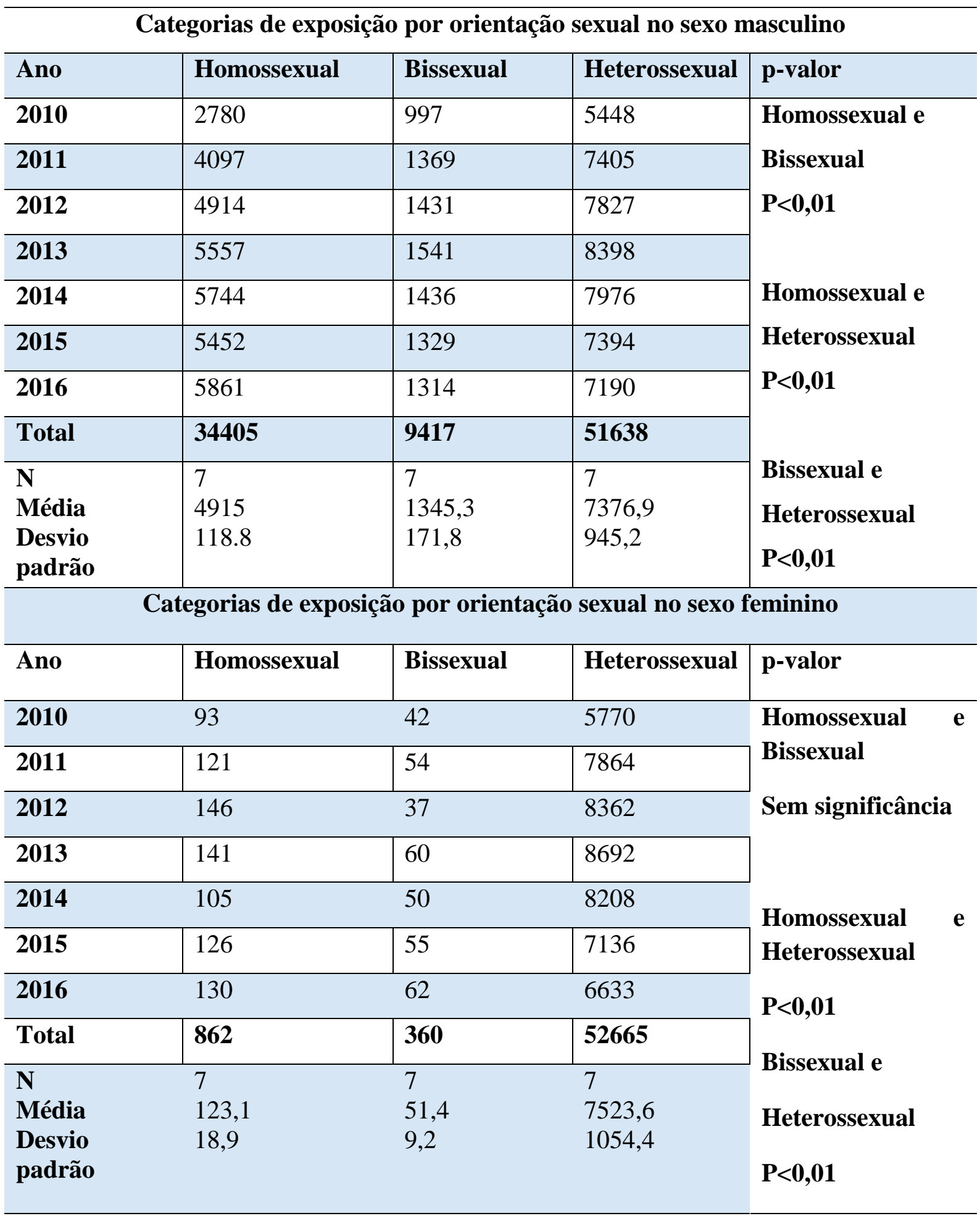

A Figura 1, abaixo, exibe o total de casos em relação ao ano, por sexo, e a respectiva linha de tendência. 


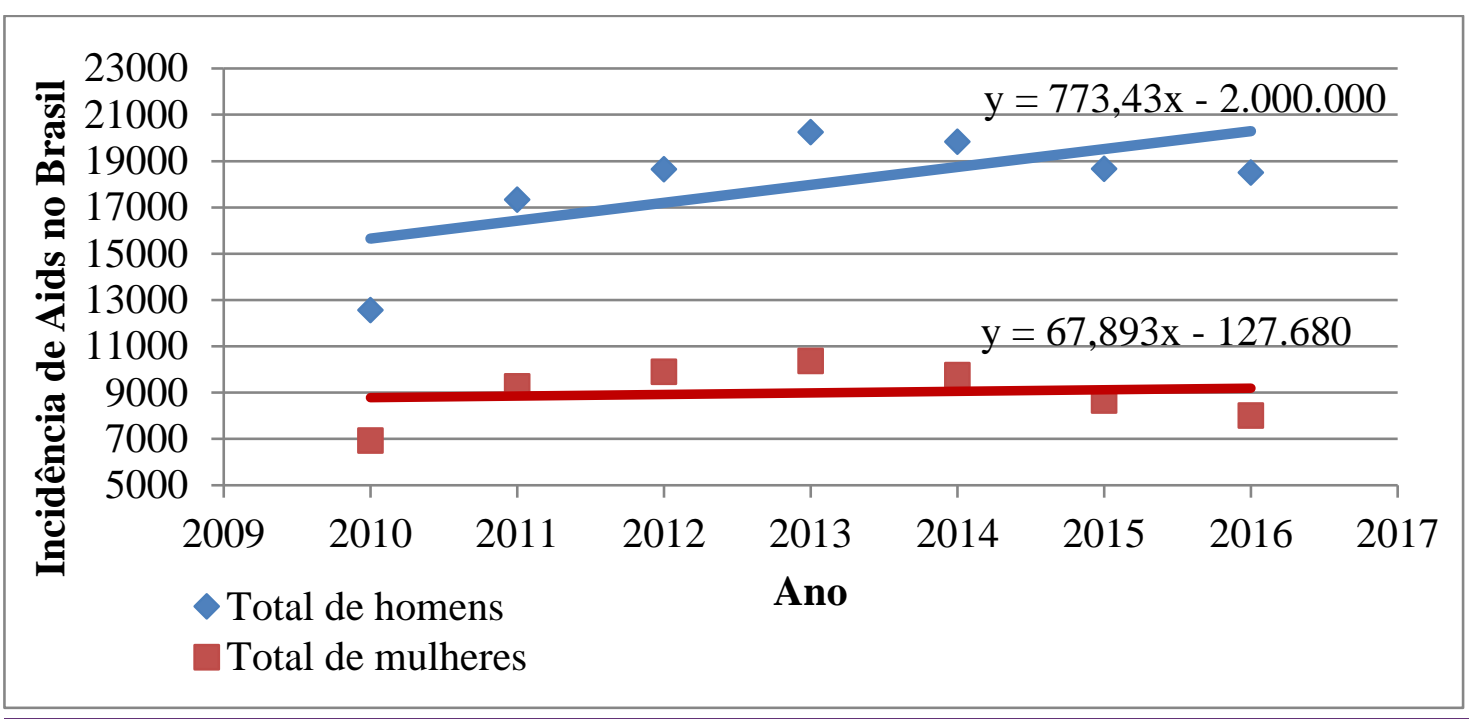

Fonte: Boletins epidemiológicos, 2017

Figura 1: Total de Casos de Aids notificados no SINAN, segundo sexo. Brasil, 2010-2016.

Do total de casos, cerca de 39\% encontram-se na região Sudeste, $19 \%$ na região Sul, 23\% na região Nordeste, $12 \%$ na região Norte e $7 \%$ na região Centro-oeste (Figura 2). Ao mesmo tempo em que o Sudeste apresenta os maiores índices, apresenta também a maior queda entre 2010 e 2016 (de mais de 17.000 para menos de 15.000).

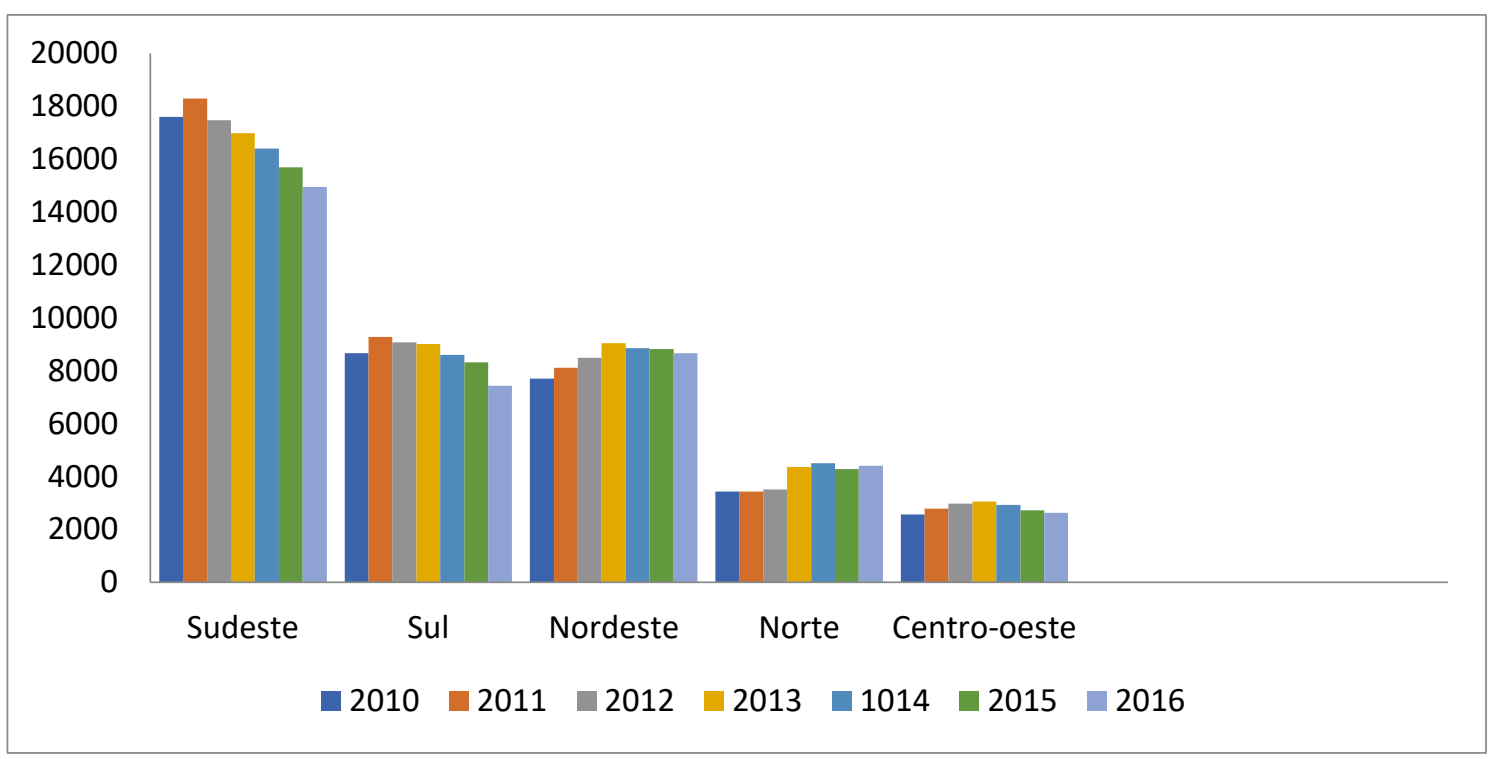

Fonte: Boletins epidemiológicos, 2017.

Figura 2: Incidência, em números absolutos(N), de Aids segundo região de residência por ano de diagnóstico. Brasil, 2010-2016. 


\section{Discussão}

Os indivíduos do sexo masculino continuam sendo os mais acometidos pela síndrome. No entanto, observa-se um crescente aumento no número dos casos de Aids notificados em mulheres. A partir da Figura 1, pode-se constatar que, apesar da redução observada nos últimos anos, a tendência é de crescimento, principalmente entre homens. De fato, estima-se que, a cada ano, ocorra um aumento de cerca de 773 novos casos entre os homens e de 68 em se tratando das mulheres. No início da epidemia, a participação feminina era mais discreta; entretanto, atualmente, a razão de incidências do histórico_chegou a 2 homens para cada mulher. Cerca de 84,0\% dessas mulheres contraíram a doença através de relações heterossexuais, já entre os homens esse percentual cai para $41 \%$.

Na África Subsaariana, as mulheres já representam 58\% da prevalência total de pessoas infectadas (MARTINS et al. 2014). Essa realidade está relacionada a fatores como a mulher ter um maior risco de contaminação, uma vez que ela recebe o sêmen masculino, que permanece em contato com a mucosa vaginal; relaciona-se também à questão do machismo ainda presente na sociedade e à desinformação, principalmente das camadas menos instruídas. Segundo Altmann et al. (2009), esta é a importância de colocar em discussão o tema de uma educação sexual à luz dos conceitos de ética, liberdade e autonomia.

Entre os anos de 2010 e 2015, 72,1\% dos casos de Aids notificados no SINAN pertenciam ao grupo de heterossexuais, sendo a maioria formada por homens, com 51,4\%. Tal fato contrasta com o início da epidemia, quando casos da doença eram diagnosticados apenas entre a população homo/bissexual. A maioria dos casos em heterossexuais é descoberta por acaso, uma vez que essa população ainda vê a Aids como uma "peste gay” e não se percebe vulnerável. Nesse interim, homossexuais soropositivos continuam sendo alvo de comorbidades associadas à violência, ao descaso na saúde pública e aos transtornos mentais.

Consoante Fernandes et al. (2017): "a imagem social de pessoas inferiores aos demais fez com que sofressem situações percebidas como ainda mais violentas, especialmente o abuso sexual, as agressões familiares e a violência institucional, praticada na escola e em nome da religião." Em meio a este contexto, a busca de apoio e compreensão foi tentada, mas o encontrado foi débil, sendo, por vezes, ofertada por profissionais como enfermeiros e psicólogos após a descoberta da infecção pelo HIV.

Embora a categoria heterossexual possua, quantitativamente, um maior número de casos registrados, a velocidade de contaminação entre homossexuais do sexo masculino está 
aumentando. Em 2010, essa categoria representava 29,5\% dos novos casos identificados em homens, chegando, no ano de 2015, ao patamar de 36,5\%. Como descrito por Jeffries et al. (2017), as relações socioambientais, socioculturais e econômicas são influências para a consolidação desta realidade: "ambientes insalubres e agressivos afetam negativamente a saúde de jovens homossexuais, bissexuais e outros homens que fazem sexo com homens, provocando isolamento e silêncio em torno da sexualidade, e colocando-os em risco de infecção pelo HIV".

A alta prevalência de violência familiar, sexual e institucional implica em mudanças na significação dos poderes interpessoais, na socialização, no autoconhecimento, no autojulgamento, nas imagens que se constroem dos espaços domésticos, institucionais e dos territórios de convívio.

A principal via de transmissão da Aids continua sendo a sexual (cerca de 80,0\% dos casos, no período analisado). A transmissão por via sanguínea é responsável por menos de 6,0\% das notificações, e o número de usuários de drogas injetáveis (UDI) infectados caiu, continuamente, devido, em especial, à adoção de práticas mais seguras na utilização dessas drogas. A transmissão do HIV por transfusão sanguínea, embora insignificante, ainda existe, mesmo com todos os processos de triagem do sangue.

Desde que se constatou a transmissão vertical da doença, tem-se conseguido controlar o número de novos casos por essa via de transmissão através da administração de antirretrovirais para a mãe e seu filho. No período estudado, esses casos representaram apenas $0,6 \%$ do total de notificações.

A maioria dos casos de Aids no Brasil encontra-se no Sudeste, principalmente nos grandes centros urbanos, mas a doença já conseguiu disseminar-se nos municípios menores espalhados por todo o território nacional. Diz-se, desse modo, que há um processo de interiorização. Ocorreu um aumento significativo no número de casos registrados na região Nordeste desde o início da epidemia. Além dos grupos acometidos inicialmente pelo HIV/AIDS, como os homossexuais, profissionais do sexo, usuários de drogas injetáveis e hemofílicos, também passaram a ser acometidas as pessoas em relacionamentos estáveis que possuem comportamentos de risco e não utilizam preservativos, os adolescentes, os idosos, os indivíduos com baixa escolaridade, renda reduzida e que habitam nas regiões interioranas do país (CUNHA et al. 2015).

A faixa etária mais acometida pela doença, desde os seus primórdios, encontra-se entre os 20 e 49 anos, principalmente na idade de 20 a 29 anos. Entre os anos de 2010 e 2015, essa perspectiva se manteve; no entanto, percebe-se um crescimento na notificação de casos entre 
as faixas de 10 a 19 anos e na faixa de 50 anos ou mais. O aumento do número de casos na população com mais de 50 anos pode ser devido à mudança no comportamento sexual da sociedade contemporânea devido ao surgimento de novas drogas que aumentam a libido. Nesses casos, a transmissão normalmente ocorre entre os casais com laços matrimoniais em consequência de relações extraconjugais e sexo sem preservativo com o companheiro(a).

O envolvimento social se revela pelo enfrentamento da disparidade, da pobreza e da discriminação, pois estas aumentam a vulnerabilidade das pessoas em relação ao HIV/Aids e dificultam o acesso à necessária prevenção, aos cuidados médicos e à adesão ao tratamento (GRECO et al. 2016).

No seu início, as pessoas infectadas eram, majoritariamente, bem instruídas, integrantes dos estratos médios e superiores da sociedade. Percebe-se, atualmente, que esse panorama sofreu alterações marcantes: a maioria dos "soropositivos" não terminaram sequer o ensino médio, o que denota a consolidação do processo de pauperização da doença se levarmos em conta a variável escolaridade para analisar esse perfil. Como agravante, pode-se citar a dificuldade que essa classe social enfrenta no tocante a informações sobre saúde, o que dificulta a prevenção. Uma vez que a educação em saúde se refere também a uma combinação de ações de políticas públicas saudáveis, criação de ambientes saudáveis, reforço da ação comunitária, desenvolvimento de habilidades pessoais, e reorientação do sistema de saúde (SALSI et al. 2013), sua carência na sociedade leva ao processo de falecimento dos meios de saúde.

\section{Conclusão}

O artigo frisa a representação epidemiológica do estereótipo da síndrome hodiernamente: homem, heterossexual, habitante dos grandes centros urbanos. A Aids continua sendo um grave problema de saúde pública no Brasil. As altas taxas anuais de infecção sugerem que o modo de abordar a doença precisa ser ampliado. A falta de orientação nas escolas e nos veículos de massa para a população que está iniciando ou já possui uma vida sexual ativa abre espaço para a disseminação do vírus.

A melhor forma de prevenção da Aids é a instrução, alertando, continuamente, a população para a necessidade da prática sexual segura e para o risco de contaminação por objetos perfurantes compartilhados, além do controle adequado de sangue e hemoderivados. Fala-se, atualmente, em possíveis vacinas; no entanto, essa possibilidade ainda parece distante. 
Nesse viés, torna-se nítida a necessidade do desenvolvimento de planejamentos governamentais atualizados a fim de enfrentar essa síndrome latente no país.

\section{Referências}

BRASIL. Boletim Epidemiológico HIV/Aids 2016. Brasília: Ministério da Saúde, 2017. 48 v.

FERNANDES, Hugo et al. Violência e vulnerabilidade ao HIV/AIDS em jovens homossexuais e bissexuais. Acta Paul Enferm, São Paulo, v. 4, n. 30, p.390-396, 07 ago. 2017.

GRECO, Dirceu Bartolomeu. Trinta anos de enfrentamento à epidemia da Aids no Brasil, 19852015. Ciência \& Saúde Coletiva, [s.l.], v. 21, n. 5, p.1553-1564, maio 2016. FapUNIFESP (SciELO). http://dx.doi.org/10.1590/1413-81232015215.04402016.

SALCI, Maria Aparecida et al. EDUCAÇÃO EM SAÚDE E SUAS PERSPECTIVAS TEÓRICAS: ALGUMAS REFLEXÕES. Texto Contexto Enferm, Florianópolis, v. 1, n. 22, p.224-230, mar. 2013.

ALTMANN, Helena; MARTINS, Carlos José. Educação Sexual: ética, liberdade e autonomia. Educar, Curitiba, Curitiba, v. 1, n. 35, p.63-80, 10 jul. 2009.

ANJOS, Rosana Maria Paiva dos. Considerações sobre a epidemiologia da AIDS. Revista da Faculdade de Ciências Médicas de Sorocaba, [S.1.], v. 1, n. 1, p. 1-5, maio 2007. ISSN 1984-4840.

CUNHA, Gilmara Holanda da et al. Quality of life of men with AIDS and the model of social determinants of health. Revista Latino-americana de Enfermagem, [s.1.], v. 23, n. 2, p.183-191, abr. 2015. FapUNIFESP (SciELO). http://dx.doi.org/10.1590/0104-1169.0120.2541.

BRASIL. MINISTÉRIO DA SAÚDE. . Boletim Epidemiológico/HIV-AIDS. Brasília: Ministério da Saúde - Secretaria de Vigilância em Saúde - Departamento de Dst, Aids e Hepatites Virais, 2015. 100 p.

BRASIL. MINISTÉRIO DA SAÚDE. (Org.). Doenças infecciosas e parasitárias. Brasília: Ministério da Saúde, 2013. 454 p.

\section{Como citar este artigo (Formato ABNT):}

FEITOSA, Pedro Walisson Gomes; OLIVEIRA, Ítalo Gonçalves Pita de; MAIA; Maria Andrezza Gomes; MARTINS, Maria Vitória Filgueira; RODRIGUES, Elisa Hellen Cruz; FELIX; Esther Barbosa Gonçalves; CÂNDIDO, Estelita Lima; FIRMINO, Paulo Renato Alves. De "Peste Gay" à Supremacia da AIDS entre Heterossexuais no Brasil. Id on Line Rev.Mult. Psic., 2018, vol.12, n.42, Supl. 1, p. 651-661. ISSN: 1981-1179.

Recebido: $14 / 11 / 2018$

Aceito: $16 / 11 / 2018$ 